Résumés des conférences et travaux

\title{
Culture et vulgarisation dans la France médiévale
}

Joëlle Ducos

\section{OpenEdition Journals}

Édition électronique

URL : https://journals.openedition.org/ashp/3077

DOI : 10.4000/ashp.3077

ISSN : 1969-6310

Éditeur

Publications de l'École Pratique des Hautes Études

Édition imprimée

Date de publication : 1 septembre 2019

Pagination : $277-280$

ISSN : 0766-0677

Référence électronique

Joëlle Ducos, "Culture et vulgarisation dans la France médiévale », Annuaire de l'École pratique des hautes études (EPHE), Section des sciences historiques et philologiques [En ligne], 150 | 2019, mis en ligne le 12 juin 2019, consulté le 06 juillet 2021. URL : http://journals.openedition.org/ashp/3077 DOI : https://doi.org/10.4000/ashp.3077 


\title{
CULTURE ET VULGARISATION DANS LA FRANCE MÉDIÉVALE
}

\author{
Directeur d'études : M ${ }^{\text {me }}$ Joëlle Ducos
}

Programme de l'année 2017-2018 : I. Nombre et figure au Moyen Âge. - II. Traductions multiples : enjeux, révisions et réception.

\section{Nombre et figure dans la France médiévale}

Les textes en langue vernaculaire utilisent nombres et chiffres dans des perspectives assez divergentes, du symbolisme au calcul pour le change ou les marchands. Par ailleurs dans le lexique français, une dénomination figure est utilisée en mathématique, terme dont le sens est évidemment opaque pour tout lecteur non spécialiste des mathématiques. Il a donc semblé utile de consacrer une partie de ce séminaire à ce qu'est le nombre au Moyen Âge, ses définitions, ses usages et l'évolution des concepts. Pour réaliser ce projet, la collaboration avec M. Stéphane Lamassé, maître de conférences à l'université Paris I et spécialiste des mathématiques marchandes à la fin du Moyen Âge, collaborateur du Dictionnaire de français scientifique médiéval, était nécessaire. Le programme de cette première année a voulu se centrer sur l'écriture des nombres, de leurs opérations et de leurs usages dans les textes médiévaux. Durant le premier semestre, nous avons essayé de dépasser l'illusion de la transparence et de la permanence du sens de ces termes autour de quelques textes permettant d'observer les tensions s'exerçant entre les héritages que ces hommes véhiculent et les transformations dont ils sont les contemporains, voire parfois les auteurs. Il était de notre objectif d'amener à entrevoir les rapports multiples que les médiévaux entretiennent avec les nombres. Il nous a donc fallu partir de l'Antiquité tardive et des usages des nombres en particulier pour la musique avec une intervention de M. Jean-Baptiste Guillaumin, maître de conférences à Sorbonne Université. Partant de la réception d'un passage du Timée, de ses commentaires, il a proposé d'observer les relations entre les rapports musicaux et les figures les représentant. La lecture des textes invitait à appréhender ces représentations de rapports et à en entendre les sonorités. Les lectures de Calcidius, Macrobe et Favonius Eulogius permettaient d'aborder la part musicale des nombres dans le cadre d'une harmonie du monde où ceux-ci peuvent être considérés sous forme de surface et de solides, reprenant là un héritage pythagoricien. Boèce dans son Institution arithmétique systématise les rapports, introduit une forme de nomenclature, et donne à chacun d'eux une dénomination qui a marqué le Moyen Âge. Mais un autre texte a eu une forte influence pour la diffusion des concepts de Boèce. Il s'agit du Liber numerorum d'isidore de Séville, texte bref que son éditeur, M. Jean-Yves Guillaumin, professeur à l'université de Besançon, a présenté en montrant comment les divisions paires et impaires des nombres y sont exposées. Mais c'est d'abord un des premiers traités d'arithmologie chrétienne, dont l'influence semble avoir été assez ponctuelle. On connaît plus tardivement, grâce aux éditions de Hanne Lange des traités cisterciens qui suivent une démarche identique, 
qui ont permis d'envisager ce qu'est l'arithmologie médiévale, avec une symbolique et une représentation du nombre beaucoup plus complexes que l'on peut croire. Après ces fondements de l'analyse du nombre pour la période médiévale et les transformations radicales se font au tournant des $\mathrm{XII}^{\mathrm{e}}$ et $\mathrm{XIII}^{\mathrm{e}}$ siècles, la conférence s'est intéressée à la fin du Moyen Âge. C'est d'abord avec une traduction d'une encyclopédie, celle de Barthélemy l'Anglais par Jean Corbechon à la demande de Charles V, que l'on a pu voir comment une traduction diffuse des concepts anciens : outre une analyse des procédés de traduction et des équivalences de termes à la fin du livre XIX qui est consacré aux mathématiques, la confrontation démontre une simplification extrême des chapitres latins, qui sont réduits avant tout à un exposé des nombres pour l'exégèse. Par contraste, l'évolution de la pensée mathématique en particulier dans la philosophie naturelle marque un écart considérable. Sabine Rommevaux-Tani, directrice de recherches au CNRS (UMR SPHERE) nous a montré par des exemples tirés de Nicolas Oresme, de Thomas Bradwardine et de Blaise de Parme, comment ont été introduits dans l'étude de la philosophie naturelle des outils mathématiques et logiques. Dans ces textes on retrouvait les termes de Boèce, mais dans un autre cadre de réception et donc de lecture. Trois grands usages des nombres ont pu être isolés dans ce champ de la pensée : 1 . ceux qui expriment des quantités, souvent pour des qualités; 2. les nombres qui pris dans un problème général sont interchangeables; 3. et ceux qui dénomment des rapports. Ainsi la denominatio chez Jordanus dans son Arithmétique est un nombre entier ou fractionnaire associé à un rapport. Un peu plus tard, Nicole Oresme, dans son De proportionibus proportionum propose de nommer les rapports irrationnels à partir des rapports rationnels. Ainsi, dans les recherches sur la proportionnalité entre les XIII ${ }^{\mathrm{e}}$ et $\mathrm{XIV}^{\mathrm{e}}$ siècles, émerge le besoin d'avoir une dénomination pour faire exister des objets qu'il est possible de manipuler. Léonard de Pise est un mathématicien héritier des traditions arabes et latines, écrivant à la fois des textes spéculatifs et pratiques. L'organisation des textes de Fibonacci, comme le Liber Abaci (1202) et la Practica Geometriae (1220) articulent les problèmes, les représentations tabulaires, l'utilisation de la mémoire par l'usage des mains et des pieds. Les fractions occupent une place importante dans l'œuvre de Léonard de Pise. Et l'exemple développé, par Marc Moyon, à partir de la construction d'un système fractionnaire original reflète les usages que ce mathématicien fait des nombres pour des raisons d'écriture et de calcul. La mise en perspective de ces textes avec d'autres traités italiens plus tardifs ( $\mathrm{XIV}^{\mathrm{e}}$ siècle) révèle comment les nombres fractionnaires ont pu faire l'objet d'appropriations différentes. Ces textes, dans lesquels la présence des calculs est bien plus importante laisse une place à l'écriture et la disposition de nombres, des quantités. Ce détour pour le latin et l'italien permet de mieux comprendre les textes mathématiques en français, leur place et leur importance. Les dernières séances du séminaire ont donc été consacrées à Nicolas Chuquet et à des lectures de problèmes en français. En 1484, Nicolas Chuquet écrit le Triparty en la science des nombres dans lequel il expose les nombres « en tant que on les peult nombrer », les proportions, les racines, la « regle des premiers ». L'analyse de ce texte favorise une compréhension des héritages précédents comme celui des algorismes, ou encore des proportions de Boèce dont il ne garde que l'essentiel. Le mot figure, qui semble apparaître avec les algorismes universitaires, désignant les chiffres de 0 à $9 \mathrm{y}$ 
trouve encore une place par exemple. Dans la perspective du séminaire, c'est dans sa partie sur l'algèbre que Nicolas Chuquet nous a semblé le plus intéressant à découvrir. C'est, en effet, le moment où il pose une certaine réflexion sur les nombres. Il y est conduit par ses choix de représentation utilisant un exposant pour les dénommer, ainsi $1^{1}$ qui correspondrait à notre $\times$ et $1^{0}$ serait 1 « et pourtant doresnavant les nombres auront .0. dessus eulx pour leur denominacion » (f. 84r). On retrouve, ici, le terme denomination des nombres. La comparaison des vocabulaires utilisés par toutes les arithmétiques pratiques en langue vulgaire nous a montré des clivages forts avec un poids relativement important des différentes traditions. Dans ces deux dernières interventions on perçoit aussi comment l'apparition de nouveaux nombres est susceptible d'interroger les usages aux textes plus anciens. À cette fin nous avons consacré une séance entière à la pratique de problèmes.

\section{Traductions multiples : enjeux, révisions et réception}

Ce thème était une invitation à envisager les textes de traductions non plus comme isolés, mais à voir comment à partir d'un même texte latin, des variations pouvaient se produire, des révisions et des modifications, qui démontent des transformations du projet de traduction, du lectorat. Malheureusement peu de séances ont pu effectivement être tenues en raison des difficultés d'accès au lieu du séminaire, souvent fermé.

Trois séances ont pu pourtant se tenir, la première pour une présentation du corpus que représentent les traductions multiples en français au Moyen Âge : signe d'une autorité, d'un intérêt particulier? C'est majoritairement le cas : le corpus des traductions de Végèce par exemple signale justement cette réfection multiple, sans qu'il y ait nécessairement des échos et des liens ente traductions. On s'est également intéressé à la diffusion de Jean Corbechon, dont la cinquantaine de manuscrits ne signifie pas nécessairement une homogénéité : traduction en 19 ou 20 livres, révisions, annotations et lectorats divers permettent de montrer ce qu'est la diffusion d'un texte très connu, sans être celui d'une autorité. Marie-Madeleine Huchet, Maître de conférences à l'UPEC, a présenté la traduction du De vetula de Richard de Fournival par Jean Le Fèvre, dont elle a fait une édition paru aux SATF en 2018, dont elle a montré l'art de gloser pour un texte dont l'original latin a particulièrement été diffusé.

La conférence a permis aussi au professeur Richard Trachsler, de l'université de Zurich, et invité par l'EPHE de faire deux interventions centrées sur les manuscrits et la réception : la première (8 mars 2018) portait sur « La Suite-Vulgate du Merlin. Le chaînon de trop » et présentait une enquête sur la tradition textuelle de la Suite-Vulgate du Merlin pour voir si sa composition était censée combler l'interstice entre la fin du Merlin de Robert de Boron et le début du Lancelot du Pseudo-Gautier Map ou devait clore le Merlin à qui, justement, manquait une véritable fin. La seconde (15 mars 2018) s'intéressait à « Paulin Paris (1800-1881), lecteur des cycles arthuriens 》 : autodidacte et enraciné dans le romantisme, il a produit une œuvre scientifique qui a mal résisté aux faits qu'allait mettre à jour la génération suivante, formée à l'École des chartes et ensuite à l'École pratique et à l'Université. En réalité, la dette des études médiévales à l'égard de Paulin Paris est immense : il a été l'un des premiers à véritablement lire les œuvres littéraires et à les traduire pour le grand public. Dans cette 
conférence, a été présenté son grand ouvrage Les Romans de la Table ronde, mis en nouveau langage et accompagnés de recherches sur l'origine et le caractère de ces grandes compositions, paru en 5 volumes entre 1868 et 1877 qui donne à lire le cycle du Lancelot-Graal et sa conception de la genèse du cycle.

Le séminaire s'est achevé par la présentation de travaux d'étudiants. 